\title{
The Smaller the Better: Designing Solar Energy Harvesting Sensor Nodes for Long-Range Monitoring
}

\author{
Malo Mabon, Matthieu Gautier (D), Baptiste Vrigneau, Mickaël Le Gentil, and Olivier Berder
}

Univ Rennes, CNRS, IRISA, France

Correspondence should be addressed to Matthieu Gautier; matthieu.gautier@irisa.fr

Received 5 April 2019; Accepted 13 June 2019; Published 15 July 2019

Guest Editor: Zoran Stamenkovic

Copyright (C) 2019 Malo Mabon et al. This is an open access article distributed under the Creative Commons Attribution License, which permits unrestricted use, distribution, and reproduction in any medium, provided the original work is properly cited.

\begin{abstract}
Emerging Low Power Wide Area Networks (LPWAN) represent a real breakthrough for monitoring applications, since they give the possibility to generate and transmit data over dozens of kilometers while consuming few energy. To further increase the autonomy of such wireless systems, the present paper proposes an original methodology to correctly dimension the key elements of an energy autonomous node, namely, the supercapacitor and the battery that mainly give the form factor of the node. Among the LPWAN candidates, LoRa is chosen for real field experiments with a custom wireless platform that proves its energy neutrality over a finite horizon. Different LoRa configurations are explored, leading to adequate dimensioning. As an example, it is shown that, for the same quality of service, the size of the solar panel needed to keep a LoRa node autonomous in the South of France is less than half of the size required in North of France.
\end{abstract}

\section{Introduction}

Future wireless standards such as the fifth generation of mobile communications are evolving to allow simultaneously high datarate transmissions for classical end-user applications such as telephony or video, very low latency for vehicle, and safety applications but also low power transmission required by the connected thing. The latter class, also known as Internet of Things (IoT), needs to accept an exponentially growing number of nodes due to a large panel of applications. While mobile operators are able to upgrade their base stations in order to deal with the IoT by allowing the $4 \mathrm{G}$ Cat-M or NB-IoT standards, several technologies dedicated to very low power consumption recently emerged in nonlicenced-ISM (Industrial Scientific and Medical) bands, among which we can cite LoRa, SigFox, or Weightless [1]. As the radio range of most of these technologies can reach several kilometers and even tens of kilometers, they are called Low Power Wide Area Networks (LPWAN).

These wireless nodes generally embed several sensors to monitor their direct environment and regularly transmit a low amount of data to a base station. Smart agriculture typically represents an application domain where LPWAN nodes are very useful, due to the huge number of nodes required to monitor fields or farm buildings and the distance to cover from remote places to the main site. Of course, the more you deploy sensors, the less you want to have to change the batteries, especially if some of them are deployed in harsh environment. Energy consumption therefore becomes a critical issue for wireless node designers, and huge efforts were recently devoted to increase node lifetime [2].

Besides, some harvesters are able to collect surrounding energy from various sources [3], e.g., light, heat, vibrations, electromagnetic waves, or even microbial fuel cell. Provided that energy managers $[4,5]$ are carefully designed, it is therefore even possible to optimize the Quality of Service (QoS) while keeping a total energy autonomy. Among all harvesters, photovoltaic panels offer from far the best power outcome and are able to efficiently transform outdoor light to feed wireless nodes [6]. However, to avoid battery failures that either could be very disadvantageous for farm monitoring or even be dangerous for the node longevity, the panels are generally oversized [7]. As they represent the element that decides the form factor of nodes, this leads to larger, more expensive, and also more visible nodes and therefore it is subject to intentional or unintentional damages. To overcome these drawbacks, the present paper proposes a methodology to reduce as much as possible the energy harvesting and 
storage elements while guarantying the desired quality of service, namely, the data rate.

Our contributions are the following:

(i) An energy autonomous node architecture with LoRa communication capabilities.

(ii) An optimization methodology for the energy harvesting and storage elements of the wireless node.

(iii) The exploration of both application requirements and light conditions and the impact on the node dimensioning.

The remainder of this paper is organized as follows. Section 2 presents related works on existing long-range oriented standards and energy harvesting. Our wireless node architecture is introduced in Section 3. Section 4 focuses on the LoRa standard and our energy consumption characterization process, followed by a experimental validation in Section 5. Finally, Section 6 concludes this paper and discusses further works.

\section{Related Works on Energy Harvesting for Long-Range Platforms}

In order to enable sustainable operation of IoT nodes, energy harvesting technologies have been considered for a long time by improving both hardware components and platforms and the associated software methods to properly manage the energy consumption $[4,8,9]$. These software components are often referred to as energy managers and are not addressed in this study. At hardware level, efforts focus on three main components of the energy harvesting system: the harvester, the energy converter, and the energy storage. Converting energy from the harvester to the storage is crucial for any energy harvesting circuit. The energy converter of the platform aims to transform the incoming energy into a suitable voltage in order to charge the energy storage (super-capacitor or battery). Different power converter architectures can be used to regulate the energy from the energy harvesting source voltage to a suitable output voltage. While the simplest architecture relies on a Low DropOut (LDO) regulator, many solutions use an SMPS (Switched Mode Power Supply) architecture in which a switch between the energy source and the energy load is rapidly opened and closed. SMPS can easily provide good conversion efficiency, from $70 \%$ to $90 \%$, can boost a low harvested voltage but are more complex and expensive than LDOs. Moreover, each energy harvesting source has an optimal operating point, called Maximum Power Point (MPP). In order to maximize harvesting efficiency, the energy converter can use MPPT (MPP Tracking) techniques in order to keep its input voltage close to the operating voltage for which the harvested power is maximized. All functions (voltage conversion, battery charging, and MPPT) are now integrated in a single Integrated Circuit (IC), such as the LTC3108 [10] from Linear Technology, the BQ25570 [11] from Texas Instruments, or the SPV1050 [12] from ST Microelectronics.
At an industrial level, few devices powered by energy harvesting are currently available and they mostly use shortrange communications [13-15]. For instance, Z-Beacon from Zolitron Technology [13] uses solar energy to power a Bluetooth 5 communication node and MVA 00X family from Micropelt [14] uses a thermoelectric generator to power an EnOcean short-range transceiver. To the best of our knowledge, Z-Node [13] is the only industrial LPWAN node powered by solar energy harvesting. It uses Sigfox [16] for its long-range communication but also includes short-range communications (Bluetooth 5 and NFC) and many sensors (temperature, acceleration, illumination, GPS, and magnetic fields). However, the problem of these devices is a lack of methodology for efficiently choosing both harvester and energy storage device according to a target quality of service. Indeed, most of them are designed for a given data and/or sensing rate. However, new LPWAN standards such as LoRa allow a wide range of data and packet rates. The node energy consumption of the node can consequently vary of several orders of magnitude from one configuration to another.

Powering LoRa based devices with energy harvesting is, as far as we know, still a research work [17-19]. Reference [17] presents an energy-efficient multi-sensing platform that exploits energy harvesting, long-range, communication and ultra-low-power short-range wake-up radio to achieve self sustainability in a kilometer range network. Energy harvesting is designed around the BQ25570 IC from Texas Instruments, which allows a conversion efficiency of $90 \%$ and provides MPPT capability. The multisource energy harvesting platform proposed in [19] relies on a circuit which switches between multiple heterogeneous energy sources and uses a single power conditioning block. A prototype has been developed and validated with a LoRaWAN platform. A single Power Management IC (PMIC) is used, the SPV1050 from ST Microelectronics, allowing a wide input voltage range. However, due the multiple energy sources, the MPPT can not be used, thus reducing consequently the harvesting efficiency.

To sum up, there is currently no commercial LoRa-based platform powered by energy harvesting but only few research works that mainly focus on the validation of the SW (energy manager) part $[18,20]$ and do not address the dimensioning of the hardware elements [7].

\section{Methodology for Dimensioning Energy Harvesting Platforms}

3.1. Platform Model. The goal of the platform studied in this paper is to measure information with several sensors and to transmit this collected data using a radio transceiver, while being powered by solar energy harvesting. The model of such a sensor node is shown in Figure 1. Two main parts can be identified: the energy harvesting part and the processing part. Firstly, the energy harvesting part is made up of the energy harvester (i.e., solar panel), the energy manager chip, a way to store the harvested energy (a supercapacitor and/or a battery), and a chip able to measure the current and voltage of the battery. Secondly, the processing part consists of a microcontroller unit (MCU) which manages both the sensors and 
TABLE 1: Input parameters description.

\begin{tabular}{lcc}
\hline Name & Unit & Description \\
\hline$I_{\text {Rad }}$ & $\mathrm{mA}$ & consumed current of the radio module in active mode \\
$I_{\text {RadSleep }}$ & $\mathrm{mA}$ & consumed current of the radio module in sleep mode \\
$I_{M C U}$ & $\mathrm{~mA}$ & consumed current of the MCU in active mode \\
$I_{M C U S l e e p}$ & $\mathrm{~mA}$ & consumed current of the MCU in sleep mode \\
ToA & $\mathrm{s}$ & Time On Air: duration of active radio \\
$T_{\text {Send }}$ & $\mathrm{s}$ & period between two transmissions \\
$T_{\text {Act }}$ & $\mathrm{s}$ & time when MCU is active for a transmission \\
$I_{\text {Sens }}$ & $\mathrm{mA}$ & consumed current of the sensor in active mode \\
$I_{S e n s S l e e p}$ & $\mathrm{~mA}$ & consumed current of the sensor in sleep mode \\
$T_{M C U S e n s}$ & $\mathrm{~s}$ & duration of active MCU for sensing operation \\
$T_{S}$ & $\mathrm{~s}$ & period between two sensing operations \\
$T_{S e n s}$ & $\mathrm{~s}$ & duration of active sensor \\
$I_{B M}$ & $\mathrm{~mA}$ & consumed current of the battery manager \\
$V_{E o C}$ & $\mathrm{~V}$ & supercapacity maximum voltage (End of Charge) \\
$V_{U V P}$ & $\mathrm{~V}$ & supercapacity minimum voltage (Under Voltage Protection) \\
$\mu_{M P P T}$ & & efficiency rate of the MPPT device \\
$n b_{W D}$ & day & number of working days for the node \\
$n b_{\bar{L} D}$ & day & number of days without harvesting \\
$T_{E H}$ & $\mathrm{~s}$ & duration with harvesting over one day \\
$T_{\overline{E H}}$ & $\mathrm{~s}$ & duration without harvesting over one day \\
\hline
\end{tabular}

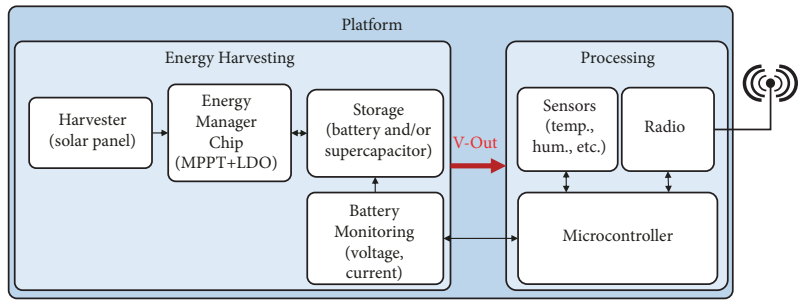

Figure 1: Platform block diagram.

a radio communication module. The proposed methodology is quite generic and does not depend on the types of radio transceiver, MCU, and sensors. Thanks to power characterization using microbenchmarks, any technology can be used in this framework. In our experimentation, the LoRa LPWAN will be addressed but other radio standards can be used. All parameters of the proposed model are given in Table 1 for the required input values and in Table 2 for the intermediate results and the outputs.

\subsection{Dimensioning Energy Harvesting Components}

3.2.1. Energy Consumption Model of the Platform. In order to dimension energy harvesting components, a duty-cycle model is proposed in which the node switches between a sleep state (the radio is turned off) and an awaken state for transmitting the data according to a periodical schedule. The time interval between two consecutive transmissions is called the wake up interval and is denoted $T_{\text {send }}$. However, to get a flexible and accurate model, two independent and distinct operations are defined: one for the radio transmission and one for the sensing. The time profiles of these operations are described in Figure 2.

Figure 2(a) introduces the cycle of a radio transmission. The MCU is firstly awaken and consumes $I_{M C U}$. It then turns on the radio module that transmits the data. The radio module creates a current peak $I_{\text {Rad }}$ during the Time on Air denoted ToA. Finally, the MCU goes back to sleep. The total time of activity is denoted $T_{A c t}$. The cycle can be split into two parts, the active and the sleep states, and the average current per each part is defined by

$$
\bar{I}_{A c t}=I_{M C U}+I_{\text {Rad }} \frac{T o A}{T_{A c t}}+I_{\text {RadSleep }}\left(1-\frac{T o A}{T_{A c t}}\right),
$$

and

$$
\bar{I}_{\text {Sleep }}=I_{\text {MCUSleep }}+I_{\text {RadSleep }} .
$$

Figure 2(b) introduces the cycle of a data sensing. First the MCU is awaken and then orders the sensor to acquire the measure. The sensing consumes $I_{\text {Sens }}$ and lasts $T_{\text {Sens }}$ while the MCU is turned on during $T_{M C U S e n s}$. However, in order to avoid considering several times the sensor current in sleep mode, it is considered equal to zero in this case. The period between two sensing operations is denoted $T_{S}$, and the average current can be computed as

$$
\begin{aligned}
\bar{I}_{S}= & I_{M C U} \cdot \frac{T_{M C U S e n s}}{T_{S}}+I_{\text {Sens }} \cdot \frac{T_{\text {Sens }}}{T_{S}} \\
& +I_{\text {SensSleep }} \cdot\left(1-\frac{T_{\text {Sens }}}{T_{S}}\right) .
\end{aligned}
$$


TABLE 2: Intermediate and output parameters description.

\begin{tabular}{|c|c|c|}
\hline Name & Unit & Description \\
\hline $\bar{I}_{\text {Act }}$ & $\mathrm{mA}$ & average consumed current of a transmission in active mode \\
\hline $\bar{I}_{\text {Sleep }}$ & $\mathrm{mA}$ & average consumed current of a transmission in sleep mode \\
\hline $\bar{I}_{S}$ & $\mathrm{~mA}$ & average consumed current of a sensing operation \\
\hline $\bar{I}_{\text {Cons }}$ & $\mathrm{mA}$ & average consumed current of a radio transmission \\
\hline $\bar{I}_{C}$ & $\mathrm{~mA}$ & total average consumed current \\
\hline$V_{S C}$ & $\mathrm{~V}$ & supercapacity voltage \\
\hline$V_{B}$ & $\mathrm{~V}$ & battery voltage \\
\hline$C_{S}$ & F & capacity of the supercapacity \\
\hline$Q_{B}$ & mA.s & electric charge of the battery \\
\hline$E_{C D}$ & $\mathrm{~mJ}$ & consumed energy during a day \\
\hline$E_{R D}$ & $\mathrm{~mJ}$ & harvested energy over one available day \\
\hline$P_{\text {Inst }}$ & $\mathrm{mW}$ & instantaneous power of the supply \\
\hline
\end{tabular}

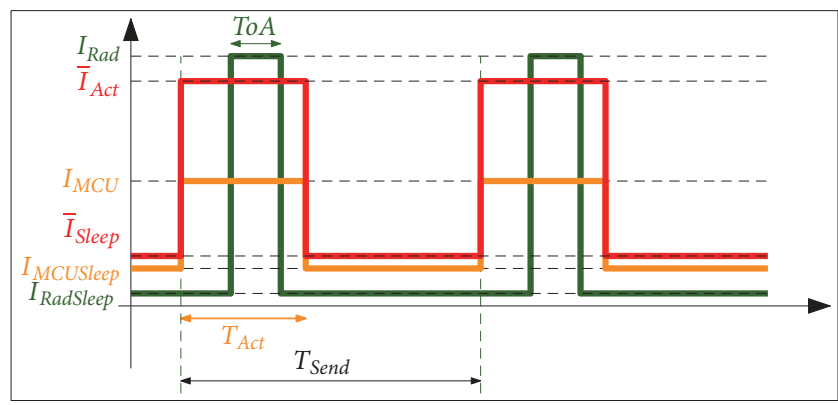

(a) Cycle of a radio transmission: MCU (orange) and radio (green) current consumptions

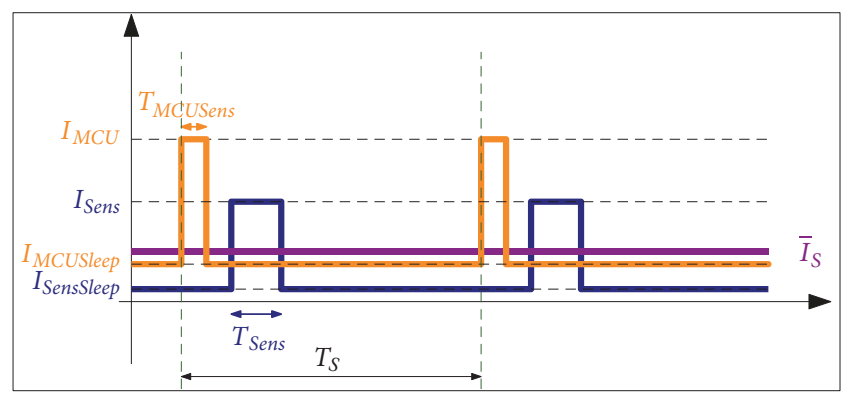

(b) Cycle of a sensing operation: MCU (orange) and sensor (purple) current consumptions

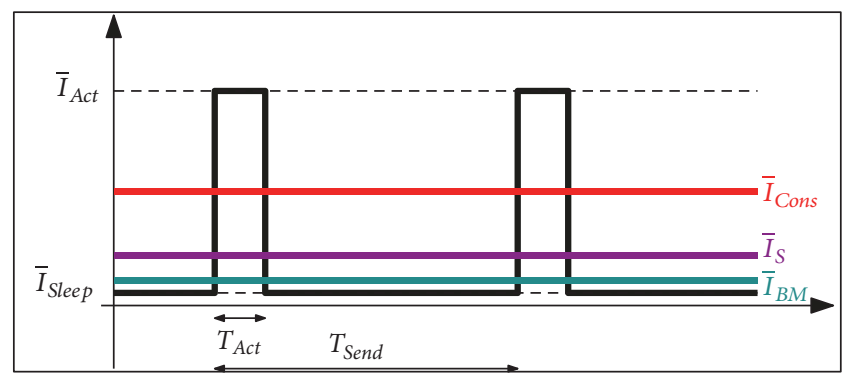

(c) Behavior of average currents

Figure 2: Current time descriptions of the cycles of both radio transmission (a) and data sensing (b) operations and the simplified cycle of the total consumption (c).

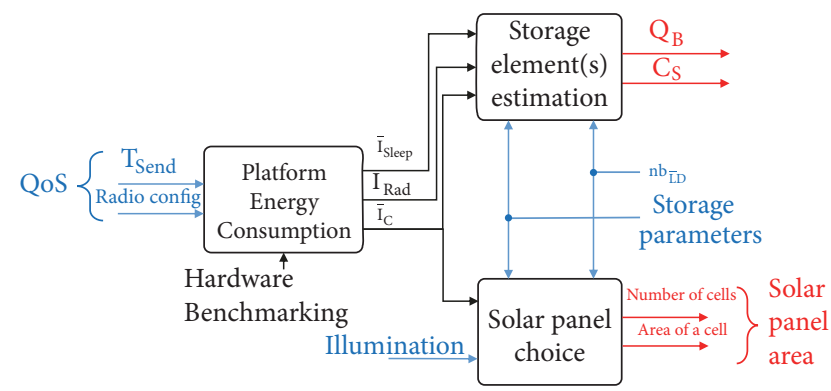

$\rightarrow$ Input
$\rightarrow$ Output
$\rightarrow$ Constant

FIGURE 3: Sizing methodology diagram.

Figure 2(c) recaps the model with the currents of both transmission and sensing cycles as well as the current consumption of the battery manager $\bar{I}_{B M}$. Then the overall current $\bar{I}_{\text {cons }}$ is defined as the mean of the sum of those three components:

$$
\bar{I}_{\text {Cons }}=\bar{I}_{\text {Act }} \cdot \frac{T_{\text {Act }}}{T_{\text {Send }}}+\bar{I}_{\text {Sleep }} \cdot\left(1-\frac{T_{\text {Act }}}{T_{\text {Send }}}\right)+\bar{I}_{S}+\bar{I}_{B M} \cdot
$$

Based on this two-step model (i.e., transmission and sensing), Figure 3 introduces the proposed methodology for choosing sizes of energy storage devices (i.e., both battery and capacitor) and harvesting components (i.e., solar panel area) of the previously introduced platform. These elements depend on QoS parameters, hardware characteristics, and environmental harvesting conditions. To this aim, the first step is to precisely control the consumed energy of the board.

Assuming that all parameters of (4) are known, the average consumed current $\bar{I}_{C}$ can be computed according to the efficiency rate $\mu_{M P P T}$ of MPPT device:

$$
\bar{I}_{C}=\frac{\bar{I}_{\text {Cons }}}{\mu_{M P P T}} .
$$




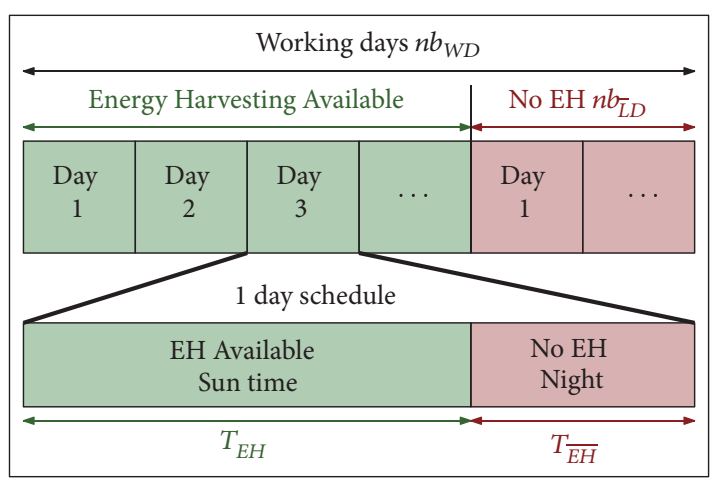

FIGURE 4: Harvesting schedule on several days and on one day.

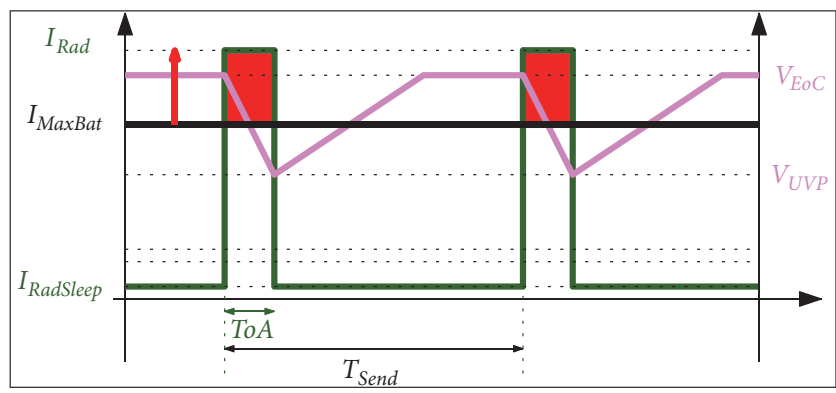

FIGURE 5: Absorption of the current peak of the radio module with capacity and battery.

3.2.2. Sizing Energy Storage Devices. The storage capacity is directly linked to both the node activity and the harvesting capabilities. The model of the solar energy harvesting considered in this work is illustrated in Figure 4 and is composed of two scales: (i) the large scale is based on the number of working days $n b_{W D}$ split into the available and no available harvesting days $\left(n b_{\bar{L} D}\right)$, for example, when a climatic event occurs like a storm; (ii) the small scale is about a day (24 hours) composed of the durations $T_{E H}$ and $T_{\overline{E H}}$ during which energy harvesting is available or not, respectively.

In our platform model, the node embeds both a battery and a supercapacitor. The battery should be able to provide the current $\bar{I}_{C}$ during $n b_{\overline{L D}}$ days or $T_{\overline{E H}}$ and leads to the inequality constraints on the battery size $Q_{B}$ :

$$
\begin{aligned}
& Q_{B} \geq \bar{I}_{C} \times 3600 \times 24 \times n b_{\bar{L} D} . \\
& Q_{B} \geq \bar{I}_{C} \times T_{\overline{E H}} .
\end{aligned}
$$

The capacitor goal is to absorb the peak of current during the transmission. The behaviors of both the current and the voltage are described in Figure 5. We assume that the battery is undersized compared to the current peak of the radio module; i.e., the maximum current provided by the battery $I_{\text {MaxBat }}$ is lower than the current $I_{\text {Rad }}$. In this case, the capacitor size is computed by considering the current variation during ToA between $I_{\text {Rad }}$ and $I_{\text {MaxBat }}$ while the capacitor voltage drops. Indeed, the storage capacitor voltage $V_{S C}$ varies between the maximum value $V_{E o C}$ corresponding to the End of Charge and the minimum value $V_{U V P}$ Under
Voltage Protection in order to avoid destruction. The worst case during the current peak is a total variation from $V_{E o C}^{C}$ to $V_{U V P}^{C}$ and leads to the constraint:

$$
C_{S} \geq \operatorname{ToA} \times \frac{I_{\text {Rad }}-I_{\text {MaxBat }}}{V_{\text {EoC }}-V_{U V P}} .
$$

The node can also be powered by using of only a supercapacitor. In this case, the supercapacitor should provide all the electric charge $Q_{B}$ (instead of only the current peak). However, for most QoS scenarii, its size will be very high (several Farads), leading to an over-prizing of the node. The cheapest solution is therefore to associate a battery and a supercapacity.

3.2.3. Sizing Solar Panel. The simplest way to calculate the solar panel size is to define the energy budget. Firstly, the average consumed energy of the system during a day is calculated:

$$
E_{C D}=\bar{I}_{C} \times V_{E o C} \times 24 \times 3600 .
$$

Knowing the number of working days and the number of days without light, the energy that has to be harvested during one available day is

$$
E_{R D}=\frac{E_{C D} \times n b_{W D}}{n b_{W D}-n b_{\overline{L D}}} .
$$

The instantaneous power of the supply is then given by

$$
P_{\text {Inst }}=\frac{E_{R D}}{T_{E H}} .
$$

The final step requires some more information related to the geographical position of the system and the solar panel. To this aim, Look-Up Tables (LUTs) of the following parameters are used:

(i) produced current density of the photo-voltaic panel $\left(\mathrm{mA} / \mathrm{cm}^{2}\right)$ as a function of the illuminance $(\mathrm{lx})$,

(ii) voltage of a photo-voltaic cell (V/cell) as a function of the illuminance $(\mathrm{lx})$,

(iii) produced current density of the photo-voltaic panel $\left(\mathrm{mA} / \mathrm{cm}^{2}\right)$ as a function of the output voltage $(\mathrm{V})$ which allows us to extract optimum voltage and current and consequently the maximum output power.

These LUTs are given by the Panasonic amorphous silicon solar cell specifications [21]. Using these LUTs, the minimum solar panel size according to the number of cells and the area of each cell for a given illumination can be computed according to the previously defined instantaneous power $P_{\text {Inst }}$.

\section{Designing Platform for LoRa Communication}

This section aims to apply the proposed methodology to design a LoRa-based platform powered by solar energy 


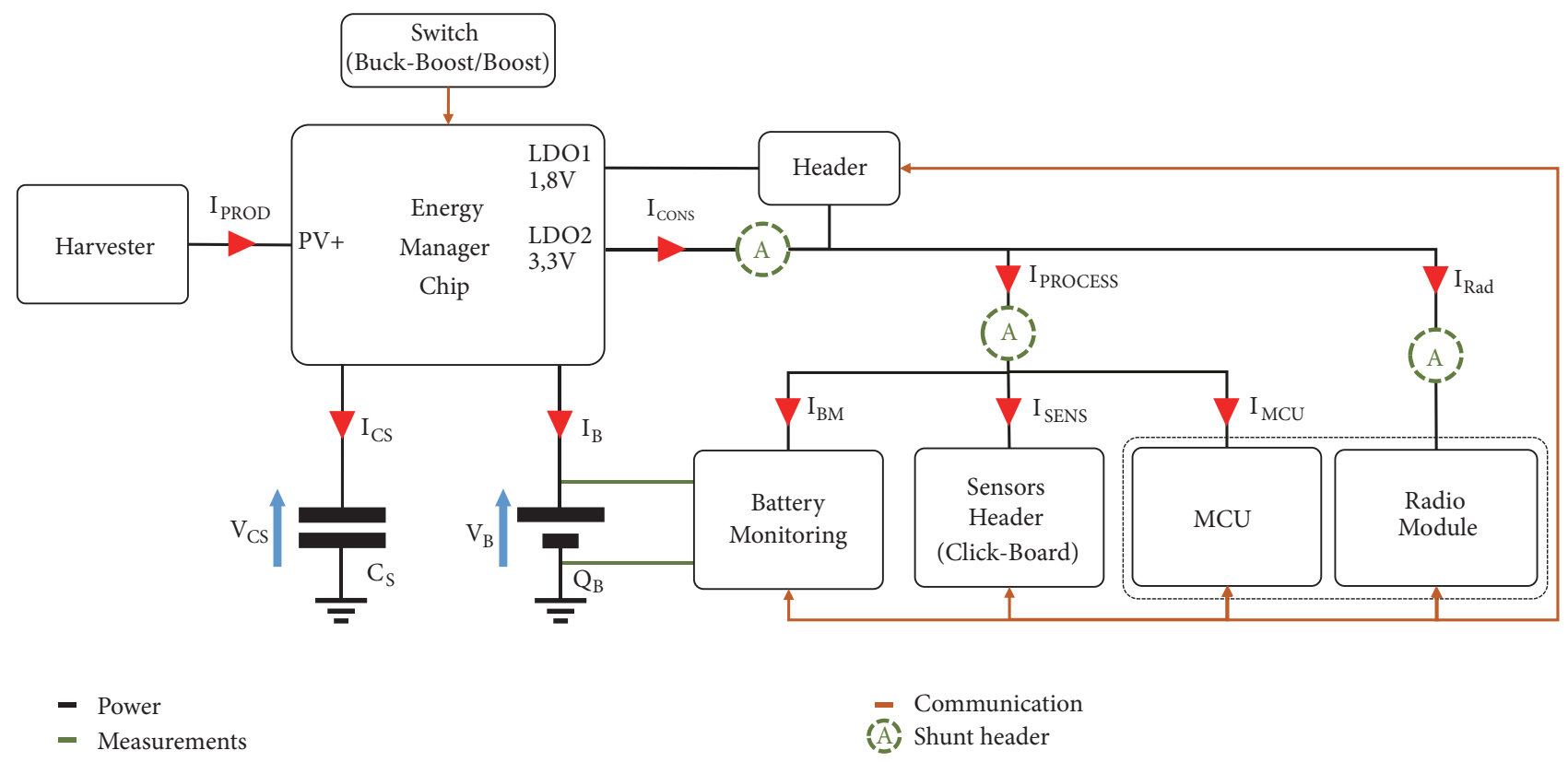

FIGURE 6: Board block diagram with energy harvesting source.

source. First, a platform has been designed using the model introduced in Section 3.1. Then, the dimensioning methodology is applied to explore the design space of the platform. As described in Figure 3, the energy consumption of the platform must be firstly characterized thanks to microbenchmarking. Then, using energy measurement values for different LoRa configurations, a design exploration is performed to see the influence of different configurations (QoS and illumination) on the hardware sizing (solar panel area and energy storage size).

4.1. Platform Design. A platform combining LoRa communication and energy has been designed and implemented using off-the-shelf components. The block diagram of the platform is given in Figure 6 while a picture of the board is shown in Figure 7. This part introduces the different blocks of both the energy harvesting part and the processing part.

4.1.1. Energy Harvesting Part. The energy harvesting part aims to supply the processing part. It is made up of the energy manager chip (SPV1050), the harvester (solar panel, Peltier module, ...), a way to store the harvested energy (a supercapacitor and/or a battery), and a chip able to measure the current and voltage of the battery.

The energy manager is implemented using the SPV1050 chip because it embeds MPPT technique, requires few external components, has a large input voltage operating range (from $150 \mathrm{mV}$ to $18 \mathrm{~V}$ ), and has a relatively low cost. However, its efficiency is a little bit lower than other PMICs having smaller input voltage operating range. A 4P2T slide switch enables changing the SPV1050 configuration from BuckBoost to Boost. With this feature, the type of harvesters (e.g., solar panels or Peltier module) can be easily changed.

For the energy storage, both previously mentioned configurations are available (i.e., using a battery and a

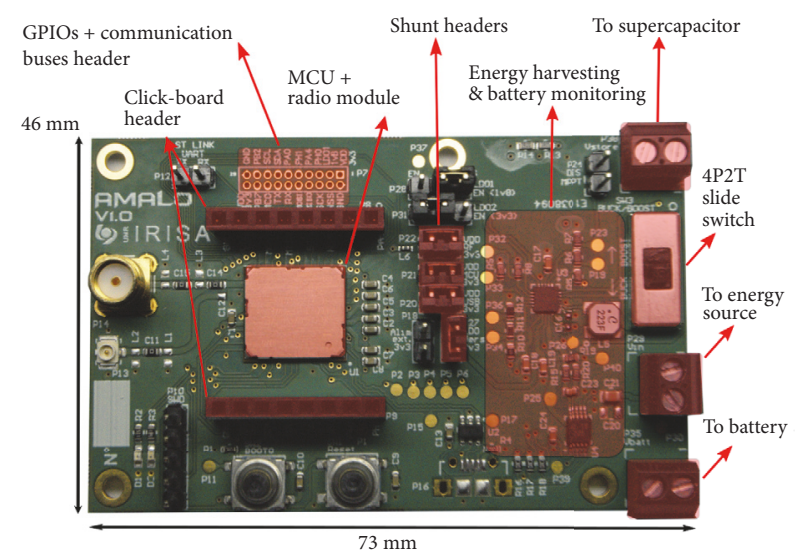

FIgURE 7: Prototype of the energy harvesting LoRa platform.

supercapacitor, or only a capacitor). Indeed, it is possible to connect a supercapacitor in order to absorb the current peaks that can occur for a transmission for instance. In addition, the SPV1050 allows the charge of any battery by configuring the protection voltages $V_{E O C}$ and $V_{U V P}$.

In order to monitor at run-time the battery state-ofcharge, the INA226 chip from Texas Instruments is implemented in the platform. It allows measuring both the battery voltage and the current and is connected to the MCU thanks to an $\mathrm{I}^{2} \mathrm{C}$ serial port. This feature allows to dynamically manage the processing block consumption by modifying the QoS for example.

4.1.2. Processing Part. The processing part allows the system to perform measurements of its environment using diverse 
sensors and to send them to a gateway using a LoRa longrange radio. This part relies on the CMWX1ZZABZ-078 chip from the ABZ Murata because it gathers a microcontroller (STM32L082CZ), a LoRa module (SX1276), and an impedance matching line on a single system-on-chip. The STM32 micro-controller is a Cortex M0+ optimized for low power applications. Its $192 \mathrm{kB}$ flash memory enables embedding both the LoRaWAN stack and the application code. In addition to the radio module, the micro-controller is connected to the sensors and to the battery monitoring chip (INA226).

The connection to sensors is achieved by dedicated ClickBoard headers or by the other pin headers with several interfaces (SPI, UART, and $\mathrm{I}^{2} \mathrm{C}$ ) and GPIOs. These interfaces are shown on Figure 7. Moreover, in order to characterize the platform by monitoring the current consumptions of the different parts, several shunt headers have been placed on the board to perform external current measurements $\left(I_{\text {CONS }}\right.$, $I_{\text {PROCESS }}$, and $\left.I_{\text {Rad }}\right)$. Figure 6 gives the block diagram of the board with the different ICs and the headers.

4.2. Energy Consumption Evaluation. Different current measurements can be performed using the shunt headers shown in Figure 6: the total current consumption of the system, the current consumption of the LoRa module, and the current consumption of the processing part (MCU, battery monitoring, and sensors). These measurements are important to characterize the different configurations of the platform that depend on the MCU modes and LoRa parameters.

Indeed, using LoRa transmission, the more range we want, the more consumption we will have. A trade-off between energy consumption and the range of the communication must be considered [22]. This trade-off is controlled by the spreading factor (SF), the bandwidth (B), the coding rate $(\mathrm{CR})$, and the transmission power $\left(P_{T x}\right)$. The CR parameter corresponds to the additional data overhead ratio incurred by the cyclic error coding to perform forward error detection and correction and takes value in the range between $4 / 8$ and $4 / 5$. The SF takes value in the range between 6 and 12 and BW between $125 \mathrm{kHz}$ and $500 \mathrm{kHz}$. The bitrate denoted $R_{b}$, for a LoRa communication, is calculated as follows:

$$
R_{b}=S F \cdot \frac{B}{2^{S F}} \cdot C R
$$

The modification of just one of the four parameters can significantly change the time on air of a LoRa communication and therefore the energy consumption. Because the space defined by these parameters is large, 3 setups corresponding, respectively, to the highest bit-rate setup (SH), the LoRa default setup (SD), and the lowest bit-rate setup (SL) were considered in this work. Table 3 shows the parameter values for each setup. Because the resulting datarates are different, the packet ToA depends on the used setup.

Figure 8 gives the different steps of the packet transmission process and the corresponding states are indicated. These steps are the ones introduced in Figure 2. The accuracy of the measurements shows that hardware and software overheads occur at the beginning and at the end of the
TABLE 3: LoRa setups used for energy measurement.

\begin{tabular}{lccc}
\hline & SH & SD & SL \\
\hline $\mathrm{CR}$ & $\frac{4}{5}$ & $\frac{4}{5}$ & $\frac{4}{8}$ \\
$\mathrm{~B}(\mathrm{kHz})$ & 500 & 125 & 125 \\
$\mathrm{SF}$ & 6 & 7 & 12 \\
$R_{b}(\mathrm{kbps})$ & 37.5 & 5.47 & 0.183 \\
\hline
\end{tabular}

TABLE 4: Current consumption of the transmission process steps.

\begin{tabular}{lcc}
\hline & Mode & Current \\
\hline \multirow{2}{*}{ Transceiver } & $I_{\text {Rad }}$ & cf. Figure 9 \\
& $I_{\text {RadSleep }}$ & $0.5 \mu \mathrm{A}$ \\
\hline \multirow{2}{*}{ MCU } & $I_{M C U}$ & $9.5 \mathrm{~mA}$ \\
& $I_{M C U S l e e p}$ & $2.8 \mu \mathrm{A}$ \\
\hline
\end{tabular}

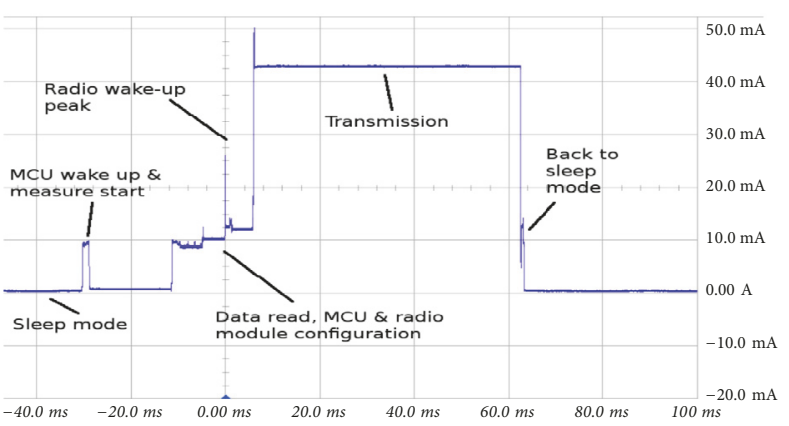

FIgURE 8: Microbenchmark of both MCU and LoRa transmission.

processes. Table 4 shows the current consumption of the most significant operation states.

The current consumption $I_{\text {Rad }}$ of LoRa transceiver is given in Figure 9 for different transmission powers $P_{T x}$. The figure shows that increasing the transmission power from $0 \mathrm{dBm}$ to $14 \mathrm{dBm}$ only doubles the current consumption. Therefore, the packet ToA will have a higher influence on the energy required for sending a packet than the transmission power. Table 5 gives the energy required for sending a 25 bytes payload using each setup and for different transmission powers $P_{T x}$. The energy required to send a packet using the SL setup is two orders of magnitude higher than the energy required to send a packet using the $\mathrm{SH}$ setup. This is due to the much lower bit-rate incurred by the lower bandwidth and the higher spreading factor, as well as the data overhead caused by a higher code rate.

4.3. Design Exploration. The experimental characterization of the proposed platform results in a fine knowledge of the current consumption for all processing steps. By combining these measurements and the QoS requirements (the method is detailed in the above Section 3), both the solar panel area and the energy storage size can be computed. This part aims to show the impact of the QoS on the hardware dimensioning. The influence of the illuminance is also evaluated.

First, the solar panel area is clearly linked to the illuminance where the node is deployed. Figure 10 explores 


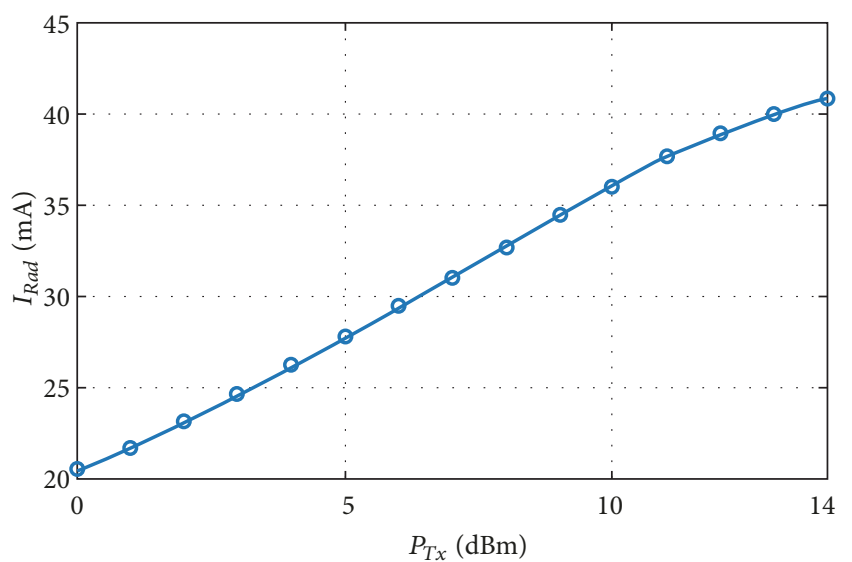

FIGURE 9: Current consumption $I_{\text {Rad }}$ of LoRa transceiver for different transmission powers $P_{T x}$.

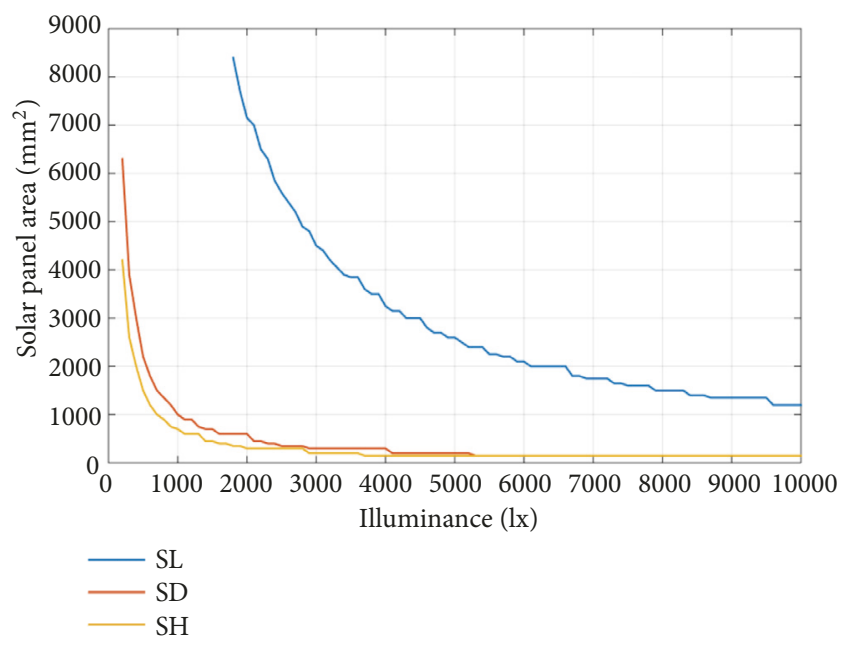

FIGURE 10: Solar panel area with respect to illuminance for different LoRa configurations.

this relation for the three LoRa setups with a $14 \mathrm{dBm}$ transmission power, a $25 \mathrm{~B}$ payload, and $T_{\text {send }}$ set to $5 \mathrm{mn}$. The illuminance ranges from $200 \mathrm{~lx}$, corresponding to minimum indoor irradiance allowed in offices to $10000 \mathrm{~lx}$, which is the maximum outdoor irradiance $[23,24]$. First, results show that the minimal solar panel area can be orders of magnitude (in $\mathrm{mm}^{2}$ ) different depending of the illuminance of the node. For instance, a $60 \mathrm{~cm}^{2}$ is required for the SD setup in an indoor deployment while only a $5 \mathrm{~cm}^{2}$ can be used in a sunny outdoor environment. The LoRa setup has also a huge impact on the solar panel area. For a 5000 lx illuminance, an area of $25 \mathrm{~cm}^{2}$ must be used for SL setup while less than $5 \mathrm{~cm}^{2}$ is required for $\mathrm{SD}$ and $\mathrm{SH}$ setups.

The impact of the QoS on the battery size is also evaluated. Figure 11 gives the minimal value of the battery needed to respect a given QoS expressed by $T_{\text {send }}$. The same configurations as for Figure 10 are used. However, another key parameter to setup the capacity is the margin needed to respect the QoS during periods of energy scarcity. This parameter is set to 2 days per week without any illuminance.
TABLE 5: Energy consumption according to LoRa configurations.

\begin{tabular}{lccc}
\hline LoRa Setup & ToA $(\mathrm{ms})$ & $P_{T x}(\mathrm{dBm})$ & Energy $(\mathrm{mJ})$ \\
\hline \multirow{3}{*}{ SH } & \multirow{3}{*}{8.99} & 0 & 0.99 \\
& & 10 & 1.45 \\
& & 14 & 1.59 \\
\hline \multirow{3}{*}{ SD } & \multirow{2}{*}{61.7} & 0 & 4.58 \\
& & 10 & 7.73 \\
& & 14 & 8.70 \\
\hline \multirow{2}{*}{ SL } & \multirow{2}{*}{1974.27} & 0 & 134.72 \\
& & 10 & 235.57 \\
& & 14 & 266.65 \\
\hline
\end{tabular}

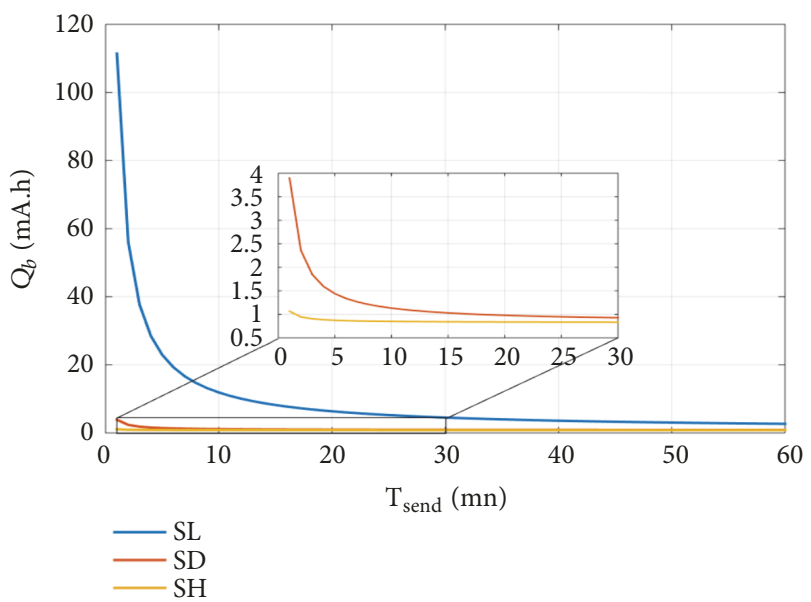

FIGURE 11: Battery sizes with respect to packet generation periods $T_{\text {send }}$ for different LoRa configurations.

Moreover, by applying (8), the supercapacitor needs to be set to $148.87 \mathrm{mF}$ in order to absorb the current peaks of the transmission process ( $40.9 \mathrm{~mA}$ for $P_{T x}$ of $14 \mathrm{dBm}$ ). Results show that increasing $T_{\text {send }}$ from $1 \mathrm{mn}$ to $60 \mathrm{mn}$ induces a battery reduction factor of 41 for SL setup, of 4.5 for SD setup and 1.3 for SH setup. The more energy the setup consumes, the higher the factor will be. Moreover, this reduction depends on a compromise between the QoS and the number of days without any illuminance.

Finally, to illustrate the benefit of a fine computation of the solar panel area, the impact of the platform localization is given in Figure 12. In this figure, the solar panel area is computed according to the minimum illuminance for each region of France. The same configurations and QoS are targeted for all regions: LoRa SL setup is used with a 14 $\mathrm{dBm}$ transmission power and a $25 \mathrm{~B}$ payload, $T_{\text {send }}$ is set to $5 \mathrm{mn}$, and the margin is set to 1 day without any light. Dimensioning results show that the solar panel area can range from $17.5 \mathrm{~cm}^{2}$ in the South of France to $44 \mathrm{~cm}^{2}$ in North of France. Therefore, an area reduction more than 2 times can be achieved to keep a LoRa node autonomous depending of its localization. 


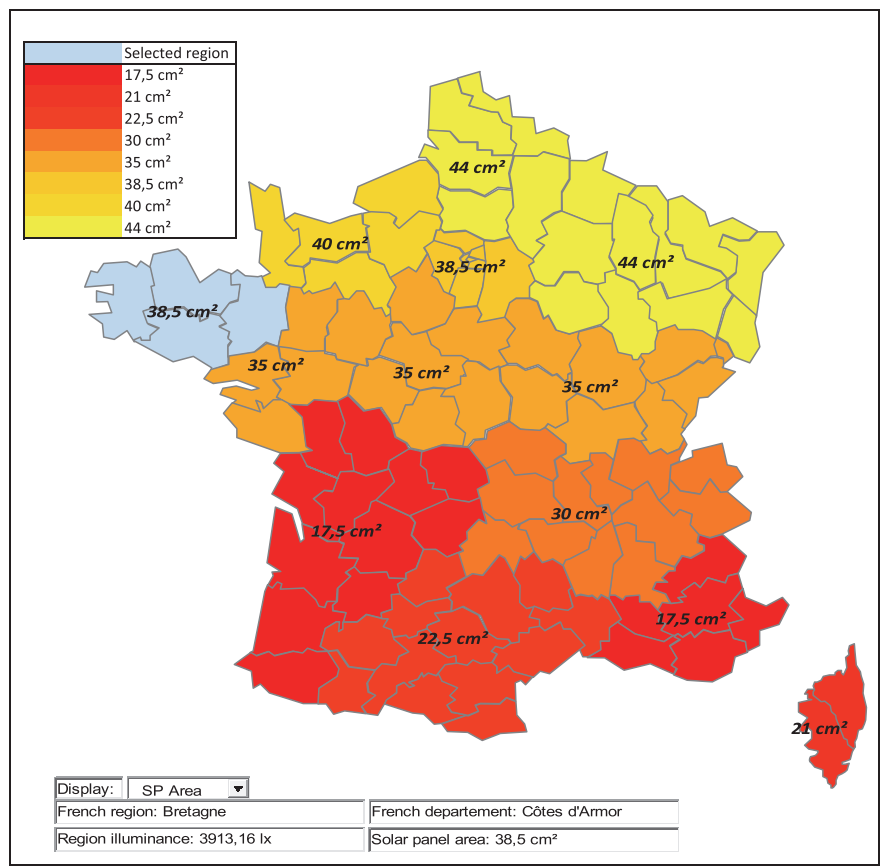

FIGURE 12: French map of solar panel area.

\section{Experimental Validation: In-Field Deployment}

5.1. Experimental Setup. The proposed methodology was applied for two different QoS and two LoRa-based platforms were implemented. Node 1 used the SL setup (i.e., spreading factor $\mathrm{SF}=12$ ) with a $14 \mathrm{dBm}$ transmission power and had a sending period $T_{\text {send }}$ of $5 \mathrm{mn}$. Node 2 used the same configuration but its SF was reduced to 10 and $T_{\text {send }}$ set to $1 \mathrm{mn}$. Node 1 has therefore a longer ToA (1974.27 ms) than Node 2 (411.65 ms) but a longer sending period. Node 1 senses temperature and humidity while Node 2 also embeds a pluviometer (inducing a negligible power consumption overhead). The battery size is set with a margin so that nodes can operate during two days without any light.

To set both solar panel area and battery size of Node 1 , Figures 12 and 11 are used as they address the same configuration. Considering a deployment in the French Britanny region, Figure 12 shows that a minimal solar panel area of $38.5 \mathrm{~cm}^{2}$ is required. Figure 11 shows that SL setup achieves $T_{\text {send }}$ of $5 \mathrm{mn}$ when a battery size of at least $26.3 \mathrm{~mA}$.h is used. Constrained by off-the-shelf components, the platform has been implemented with a $40.7 \mathrm{~cm}^{2}(7.4 \times 5.5 \mathrm{~cm})$ solar panel, a $35 \mathrm{~mA}$.h LiPo battery, and a $220 \mathrm{mF}$ supercapacity from Murata. For Node 2, its configuration was chosen in order to achieve the same hardware dimensioning.

For validation purpose, both nodes were deployed on the roof of the laboratory building, as shown in Figure 13. The nodes were deployed under the same illuminance conditions. The gateway was installed in an office room and at around $20 \mathrm{~m}$ far from the nodes. The range was not considered in this experimentation to evaluate only the impact of the energy harvesting on the QoS. The gateway was battery powered to

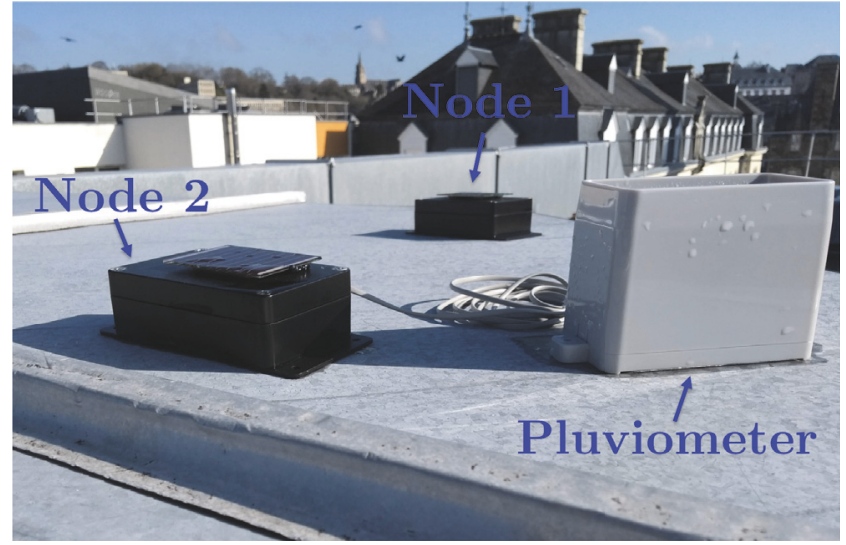

FIGURE 13: Photography of the two nodes deployed on the building roof.

gather data from both nodes, i.e., the 25B payload composed of all sensed values and the battery state-of-charge. The experiment lasted 15 days from March $8^{\text {th }}$ to March $22^{\text {nd }}$.

5.2. Experimental Results. The values from the sensors show that the weather was quite favourable during these days. The average temperatures were 21.1 degrees during days and 8.2 degrees during nights for Node 1; 20.7 degrees during days and 8.35 degrees during nights for Node 2. The pluviometer of Node 2 measured no rain precipitation over experimentation. These values are higher than the normal ones for this season: 11.5 degrees during days, 5.4 degrees during nights, and 95.7 $\mathrm{mm}$ of precipitation. The illuminance was also very high compared to the value used to set the solar panel area. 


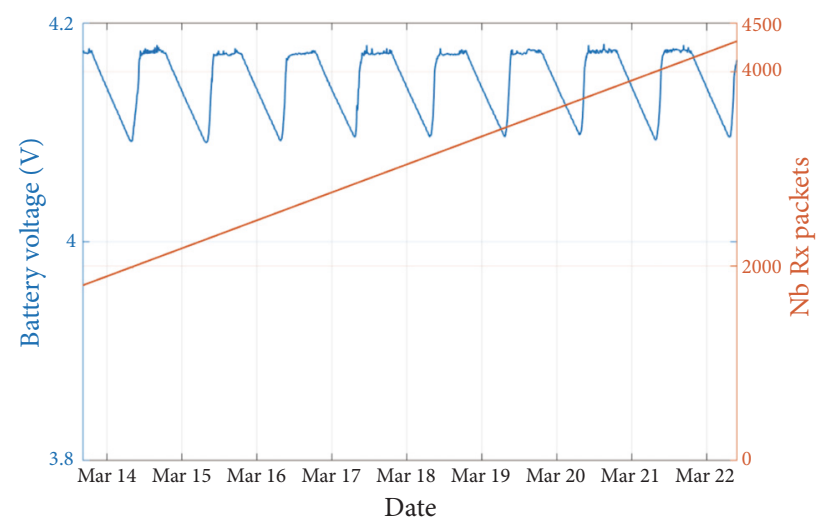

(a) Node 1

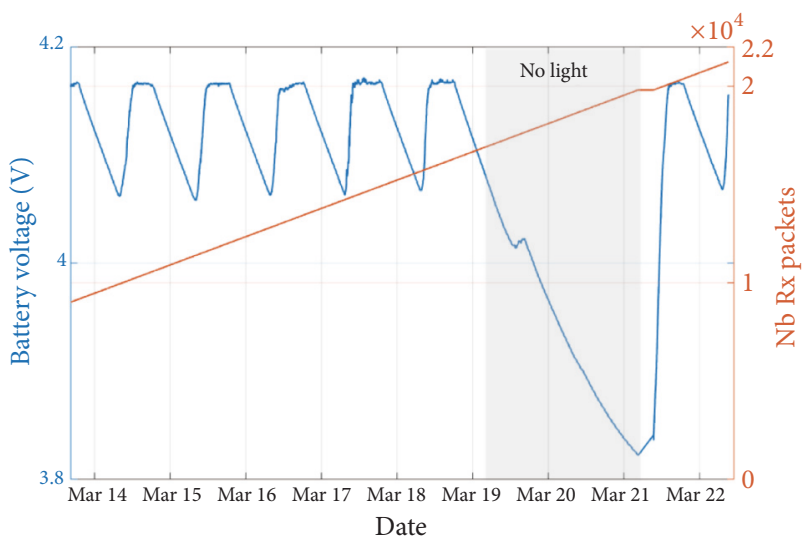

(b) Node 2

Figure 14: Battery voltage and frame counter for the two nodes.

Figure 14 shows the obtained results. The battery state-ofcharge and the frame counter are plotted for the last 9 days. Figure 14(a) gives the results for Node 1 with the lowest QoS $\left(T_{\text {send }}=5 \mathrm{mn}\right.$ ) and Figure 14(b) the results for Node 2 with the highest QoS $\left(T_{\text {send }}=1 \mathrm{mn}\right)$. Results show that both nodes operate without any packet loss during the 15 days, reaching a total of 4308 packets for Node 1 and 21233 packets for Node 2 . In order to evaluate the robustness against periods of energy scarcity, Node 2 was turned upside down at the beginning of March $19^{\text {th }}$ so that it does not harvest any energy. In these conditions, Node 2 still operates during more than two days, as expected, showing the good sizing of the battery. After the first packet losses, the node was powered again and quickly reached its periodic behavior.

\section{Conclusion}

LPWAN technologies represent a real opportunity for applications requiring both long-range and low power consumption, such as environmental monitoring or connected farms. We proposed in this paper an energy autonomous wireless node platform leveraging LoRa technology. Optimizing the size of the energy storage and harvesting elements of the platform is of prime importance, because it will directly impact the form factor of the global platform. On the one hand, to deal with the distance to the base station and channel impairments, LoRa offers several configurations by properly tuning the spreading factor or the error correcting code, but this dramatically changes the time-on-air, therefore leading to very different energy consumption for transmitting the same amount of data. On the other hand, the climate of the area where nodes are deployed also means very different light conditions. Thanks to hybrid energy modelling including accurate benchmarking on our platform, a methodology was proposed to optimize the key elements of the wireless node, ensuring application requirements while taking into account node environment. An exploration was driven to highlight the impacts of both time-on-air and illuminance on this sizing. As a toy example, it was shown that, for the same quality of service, the size of the solar panel has to be twice in the North of France than in the South, and this ratio is directly linked to the size of the whole wireless sensor node.

\section{Data Availability}

No data were used to support this study.

\section{Conflicts of Interest}

The authors declare that there are no conflicts of interest regarding the publication of this paper.

\section{Acknowledgments}

This work has been conducted in the context of the AAP PME ALAMO project labelled by the "Pôle Images \& Réseaux" and funded by "Région Bretagne" and "Lannion Trégor Communauté".

\section{References}

[1] M. Centenaro, L. Vangelista, A. Zanella, and M. Zorzi, "Longrange communications in unlicensed bands: The rising stars in the IoT and smart city scenarios," IEEE Wireless Communications Magazine, vol. 23, no. 5, pp. 60-67, 2016.

[2] F. A. Aoudia, M. Gautier, M. Magno, M. L. Gentil, O. Berder, and L. Benini, "Long-short range communication network leveraging LoRa ${ }^{\mathrm{TM}}$ and wake-up receiver," Microprocessors and Microsystems, vol. 56, pp. 184-192, 2018.

[3] R. J. M. Vullers, R. V. Schaijk, H. J. Visser, J. Penders, and C. Hoof, "Energy harvesting for autonomous wireless sensor networks," IEEE Journal of Solid-State Circuits, vol. 2, no. 2, pp. 29-38, 2010.

[4] A. Kansal, J. Hsu, S. Zahedi, and M. B. Srivastava, "Power management in energy harvesting sensor networks," $A C M$ Transactions on Embedded Computing Systems, vol. 6, no. 4, article 32, 2007.

[5] T. N. Le, A. Pegatoquet, O. Berder, O. Sentieys, and A. Carer, "Energy-neutral design framework for supercapacitorbased autonomous wireless sensor networks," ACM Journal on Emerging Technologies in Computing Systems, vol. 12, no. 2, pp. 1-19, 2015.

[6] M. A. Green, Y. Hishikawa, W. Warta et al., "Solar cell efficiency tables (version 50)," Wiley Journal of Progress in Photovoltaics: Research and Applications, vol. 25, no. 7, pp. 668-676, 2010. 
[7] W. Lee, M. J. Schubert, B. Ooi, and S. J. Ho, "Multi-source energy harvesting and storage for floating wireless sensor network nodes with long range communication capability," IEEE Transactions on Industry Applications, vol. 54, no. 3, pp. 2606-2615, 2018.

[8] F. Ait Aoudia, M. Gautier, and O. Berder, "RLMan: an energy manager based on reinforcement learning for energy harvesting wireless sensor networks," IEEE Transactions on Green Communications and Networking, vol. 2, no. 2, pp. 408-417, 2018.

[9] F. A. Aoudia, M. Gautier, and O. Berder, "Fuzzy power management for energy harvesting Wireless Sensor Nodes," in Proceedings of the ICC 2016 - 2016 IEEE International Conference on Communications, pp. 1-6, Kuala Lumpur, Malaysia, May 2016.

[10] I. Analog Devices, "LTC3108 - Ultralow voltage step-up converter and power manage," http://www.analog.com/en/products/power-management/energy-harvesting/ltc3108.html.

[11] T. I. Inc., "BQ25570 - ultra low power harvester power management IC with boost charger, and nanopower buck converter," http://www.ti.com/product/bq25570.

[12] STMicroelectronics, "SPV1050 - Ultra low power energy harvester and battery charger with embedded MPPT and LDOs," https://www.st.com/en/power-management/spv1050.html.

[13] Zolitron, "Z-Node: World's first and only autonomous cognitive sensor," https://zolitron.com/our-products/zolitron-z-node/.

[14] Micropelt, "Self-powered radiator valve," http://www.micropelt.com/fileadmin/user_upload/_PDF_MVA004_datasheet_ 1DSMVA004_0817v1le.pdf.

[15] Trameto, "Delivering any-many-multi source, micro-energy harvesting," https://trameto.com/technology/.

[16] C. Goursaud and J. M. Gorce, "Dedicated networks for IoT: PHY / MAC state of the art and challenges," EAI Endorsed Transactions on Internet of Things, vol. 1, no. 1, p. 150597, 2015.

[17] M. Magno, F. A. Aoudia, M. Gautier, O. Berder, and L. Benini, "WULoRa: An energy efficient IoT end-node for energy harvesting and heterogeneous communication," in Proceedings of the 20th Design, Automation and Test in Europe, DATE 2017, pp. 1528-1533, Lausanne, Switzerland, March 2017.

[18] F. Wu, J. Redoute, and M. R. Yuce, "WE-Safe: a self-powered wearable IoT sensor network for safety applications based on LoRa," IEEE Access, vol. 6, pp. 40846-40853, 2018.

[19] P.-D. Gleonec, J. Ardouin, M. Gautier, and O. Berder, "Architecture exploration of multi-source energy harvester for IoT nodes," in Proceedings of the 2016 6th Annual IEEE Online Conference on Green Communications, OnlineGreen Comm 2016, pp. 27-32, November 2016.

[20] P. Gleonec, J. Ardouin, M. Gautier, and O. Berder, "A realworld evaluation of energy budget estimation algorithms for autonomous long range IoT nodes," in Proceedings of the 2018 25th International Conference on Telecommunications (ICT), pp. 561-565, Saint-Malo, France, June 2018.

[21] Panasonic, "Amorphous Silicon Solar Cells Amorphous Photosensors," https://www.panasonic-electric-works.com/cps/rde/ xbcr/pew_eu_en/ca_amorton_solar_cells_2018_en.pdf.

[22] X.-C. Le, B. Vrigneau, M. Gautier, M. Mabon, and O. Berder, "Energy/Reliability Trade-off of LoRa Communications over Fading Channels," in Proceedings of the 25th International Conference on Telecommunications, ICT 2018, pp. 544-548, France, June 2018.

[23] Y. Xie, M. Sengupta, and M. Dooraghi, "Assessment of uncertainty in the numerical simulation of solar irradiance over inclined PV panels: New algorithms using measurements and modeling tools," Solar Energy, vol. 165, pp. 55-64, 2018.

[24] National Renewable Energy Laboratory (NREL), http://www .nrel.gov/. 


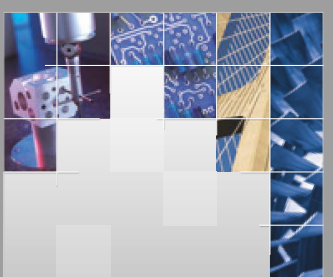

\section{Enfincering}
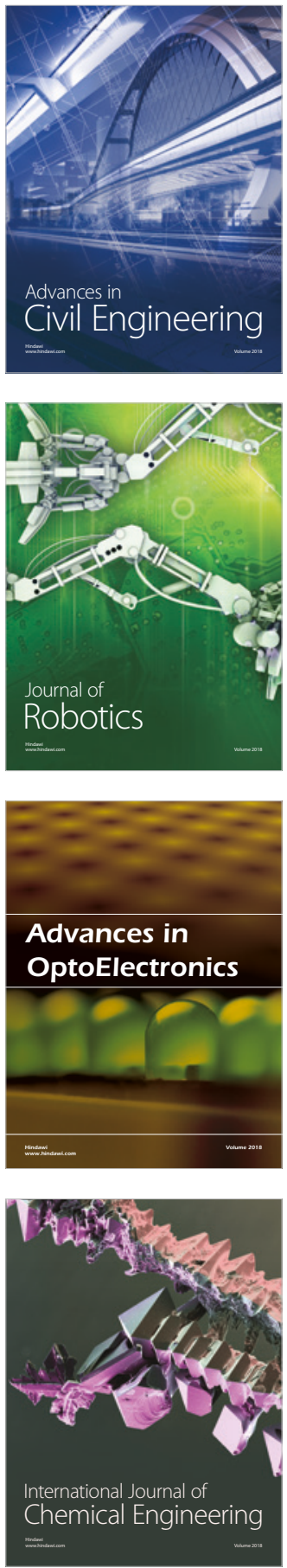

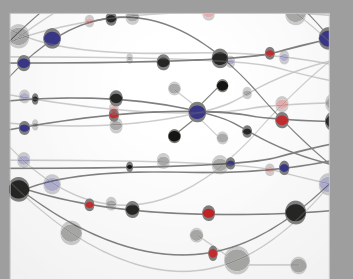

\section{Rotating \\ Machinery}

The Scientific World Journal

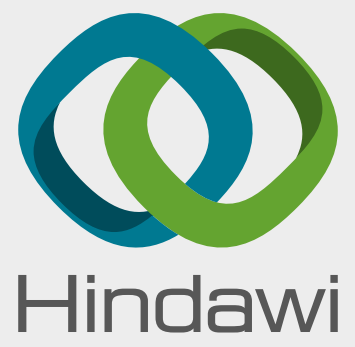

Submit your manuscripts at

www.hindawi.com
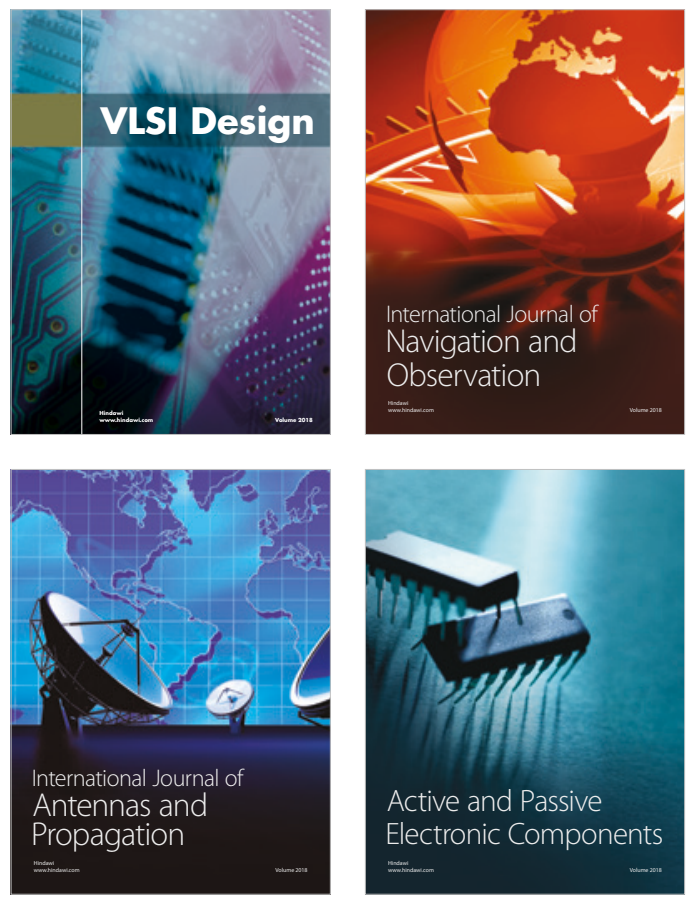
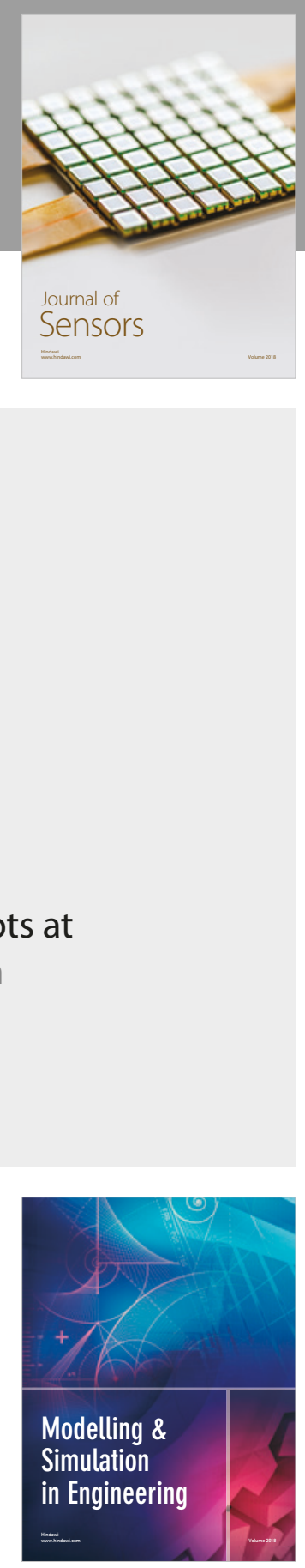

\section{Advances \\ Multimedia}
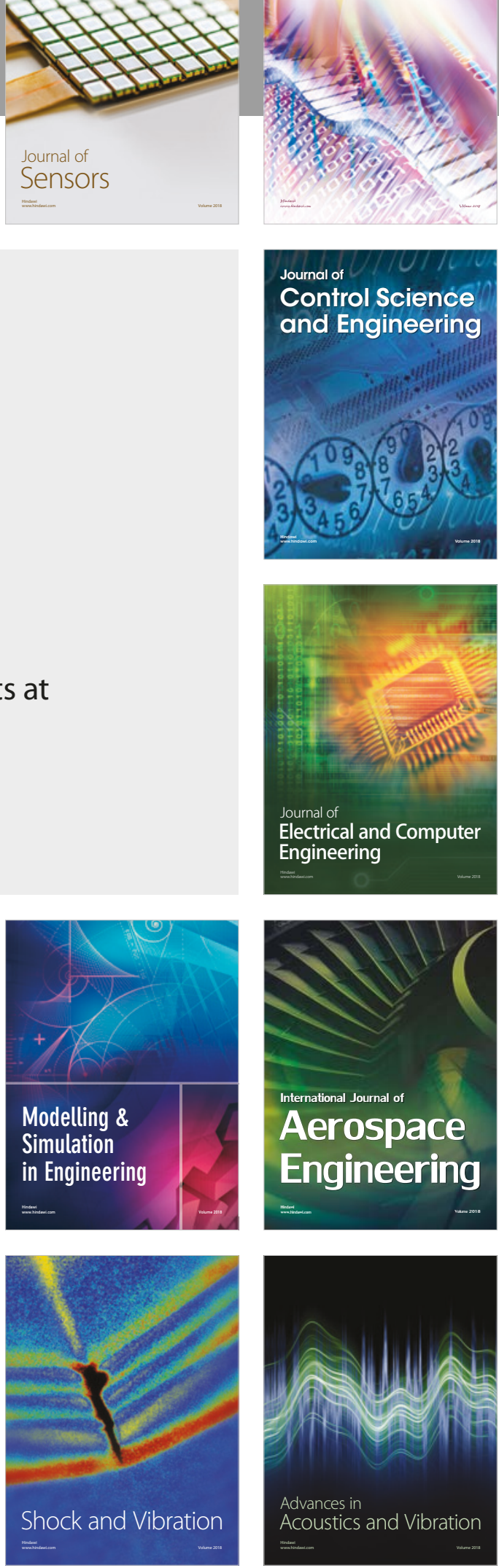